\title{
Dynamic changes in basal lamina fenestrations in rat intestinal villous epi- thelium under high-fat diet condition
}

\author{
Keisuke Morita ${ }^{1,2}$, Rie Azumi ${ }^{1}$, Masatoshi Sato ${ }^{1,2}$, Yusuke Mizutani ${ }^{2}$, Manabu HaYatsu $^{2}$, Shuji Terai ${ }^{1}$, and \\ Tatsuo UshiKi ${ }^{2}$ \\ ${ }^{1}$ Division of Gastroenterology, Niigata University Graduate School of Medical and Dental Sciences, Niigata, Japan and ${ }^{2}$ Division of \\ Microscopic Anatomy, Niigata University Graduate School of Medical and Dental Sciences, Niigata, Japan
}

(Received 25 December 2019; and accepted 17 January 2019)

\begin{abstract}
The basal lamina of the villous epithelium in the small intestine has numerous fenestrations, which are produced by leukocytes for their intraepithelial migration. We previously showed that these fenestrations change due to the dynamics of migrating leukocytes in response to dietary conditions and suggested the possibility that this change is related to the regulation of the absorption of large-sized nutrients such as chylomicrons. The present study was, thus, designed to investigate structural changes in basal lamina fenestrations in response to a high-fat diet. The ultrastructure of the intestinal villi in the rat upper jejunum was investigated by electron microscopy of tissue sections in both the normal and the high-fat diet groups, and the fenestrations in the villous epithelium of rat upper jejunum were studied by scanning electron microscopy of osmium macerated/ ultrasonicated tissues. The present study showed that free cells adhering to the fenestrations increased in the upper jejunum two hours after feeding high-fat diet and the size of the fenestrations in this region also increased after feeding high-fat diet for 2 days. This enlargement of fenestrations may play an important role in increasing the efficiency of lipid absorption by facilitating the movement of chylomicrons from the intercellular space to the lamina propria.
\end{abstract}

Absorptive epithelial cells in the small intestine are important for the absorption of nutrients. Since these cells are sealed by tight junctions on the apical side, nutrients are first absorbed from the intestinal lumen into the epithelial cells after intraluminal and membranous digestion to small molecules (19). Among these nutrients, carbohydrates and proteins are absorbed from the luminal membrane of the epithelial cells as monosaccharides and amino acids, respectively, and are released from the basolateral side (15). Neutral fats are also absorbed into the epithelial cells as monoglycerides and fatty acids, resynthe-

Address correspondence to: Prof. Tatsuo Ushiki, Division of Microscopic Anatomy, Niigata University Graduate School of Medical and Dental Sciences, Asahimachidori 1, Chuo-ku, Niigata 951-8510, Japan

Tel: +81-25-227-2062, Fax: +81-25-224-1767

E-mail: t-ushiki@med.niigata-u.ac.jp sized into triacylglycerol in the cells, and released from the basolateral side as chylomicrons (3, 13, 14). These nutrients, released into the intercellular space of the epithelium, finally accumulate in blood capillaries and initial lymphatics of the lamina propria after passing through the epithelial basal lamina. Thus, the basal lamina is considered as a barrier for these nutrients as they pass from the epithelium to the lamina propria.

Morphologically, the basal lamina of the intestinal epithelium has been directly observed by scanning electron microscopy (SEM) after removal of the epithelial cells $(8,11,16,17)$. These studies have reported the presence of fenestrations in the basal lamina, which are probably produced by intraepithelial migration of free cells (e.g., lymphocytes, eosinophils, and macrophages) from the lamina propria. On the other hand, some other studies have reported that chylomicrons pass through these fenestrations 
because chylomicron particles are too large (ca. $100 \mathrm{~nm}$ to $1 \mu \mathrm{m}$ in diameter) to pass through the basal lamina without any interruptions (14). Recently, we showed that the basal lamina fenestrations changed through dynamics of migrating leukocytes under fasted and fed states and this change may be related to the regulation of nutrient absorption, particularly that of lipid absorption (1).

To clarify the role of basal lamina fenestrations in response to changes in diet, the present study was designed to examine the morphological changes in the basal lamina fenestrations in response to a highfat diet. Using SEM after removal of villous epithelium, we observed the fenestrations of epithelial basal lamina in the upper jejunum of the animals fed either normal or a high-fat diet and compared the size and number of fenestrations between the two groups. Here, we propose that the basal lamina fenestrations may play an important role in increasing the efficiency of lipid absorption by facilitating the movement of chylomicrons from the intercellular space to the lamina propria.

\section{MATERIALS AND METHODS}

This study was carried out in accordance with guidelines for animal experiments at Niigata University Animal Experimental Guidelines after approval by the Ethics Committee of Niigata University Animal Experimentation (No. 340-2).

Animals. Male Wistar rats (Japan SLC, Inc. Shizuoka, Japan), 10 to 11 weeks old, were used in this study. They were divided into four groups, each of which consisted of three rats (Fig. 1). Group A was fed ordinary pellets (Rodent Lab Diet EQ; PMI Nutrition International Inc., Brentwood, MO, USA) for $2 \mathrm{~h}$ after fasting for $20 \mathrm{~h}$, and group B was fed high-fat pellets for $2 \mathrm{~h}$ after fasting for $20 \mathrm{~h}$. The high-fat pellets were prepared by immersing in corn oil (Cat. No. 032-17016; FUJIFILM Wako Pure Chemical Co, Osaka, Japan). Group C was fed ordinary pellets for $2 \mathrm{~d}$ after fasting for $20 \mathrm{~h}$, and group D was fed high-fat pellets for $2 \mathrm{~d}$ after fasting for $20 \mathrm{~h}$. All animals were anesthetized by intraperitoneal injection with pentobarbital sodium and were then perfused through the heart with physiological saline followed by fixation in $4 \%$ paraformaldehyde (in $0.1 \mathrm{~mol} / \mathrm{L}$ phosphate buffer). After perfusion fixation, the upper jejunum was excised from the body and immersed in the same fixative overnight.

Light microscopy and SEM for section studies. The upper jejunum was cut into small pieces (about $2 \times 1 \mathrm{~mm}$ in size) and fixed in $2 \%$ glutaraldehyde (in $0.1 \mathrm{~mol} / \mathrm{L}$ phosphate buffer) for $4 \mathrm{~h}$ followed by immersion fixation with $1 \%$ osmium tetroxide for $90 \mathrm{~min}$. They were dehydrated using a graded ethanol series and embedded in Epon 812. Semithin sections (about $1 \mu \mathrm{m}$ thick) were cut using a diamond knife, stained with toluidine blue, and observed under an optical microscope (ECLIPSE E 600; Nikon, Tokyo, Japan).

In order to observe the ultrastructure of the samples, semithin sections observed under light microscopy were further stained with uranyl acetate and lead citrate, coated slightly with platinum and palladium in a magnetron sputtering coater, and observed in a back-scattered electron (BSE) mode under a scanning electron microscope (SU-3500; Hitachi, Tokyo, Japan). This technique is useful for observing wide areas of the semithin sections at a high magnification (7).

Transmission electron microscopy (TEM) of ultrathin sections. Ultrathin sections (60-80 nm thick) were also made from the Epon-embedded samples with a diamond knife; they were stained with uranyl acetate and lead citrate and observed under a transmission electron microscope (H-7650; Hitachi).

SEM analysis of osmium-macerated/ultrasonicated tissues. The technique used in the present study was almost the same as described previously (1). The upper jejunum was further fixed in $2 \%$ glutaraldehyde (in $0.1 \mathrm{~mol} / \mathrm{L}$ phosphate buffer) for $2 \mathrm{~d}$. The transverse slices (about $2 \mathrm{~mm}$ thick) of the jejunum were made with a razor blade, washed with the phosphate buffer, and macerated in $2 \%$ osmium tetroxide at $20^{\circ} \mathrm{C}$ for $3 \mathrm{~d}$. After rinsing in the same buffer, the slices were treated in an ultrasonic bath (Bransonic Ultrasonic Cleaner; Branson Ultrasonics, Emerson Japan, Atsugi, Japan), which enabled the removal of epithelial cells from the intestinal villi. After confirming complete removal of epithelial cells under a stereomicroscope, the slices were conductive stained by treating them with $1 \%$ tannic acid solution for $2 \mathrm{~h}$, followed by a wash step in the phosphate buffer for $30 \mathrm{~min}$, and incubation in 1\% osmium tetroxide solution for $2 \mathrm{~h}$. They were dehydrated with an ascending ethanol series $(70 \%, 80 \%$, $90 \%$, and $95 \%$ for $30 \mathrm{~min}$ each, $100 \%$ for $30 \mathrm{~min}$, three times) and dried in a critical point drier (CPD2; Hitachi) with $\mathrm{CO}_{2}$. The dried specimen was mounted on an aluminum stub with double-sided tape, coated with platinum-palladium using a mag- 


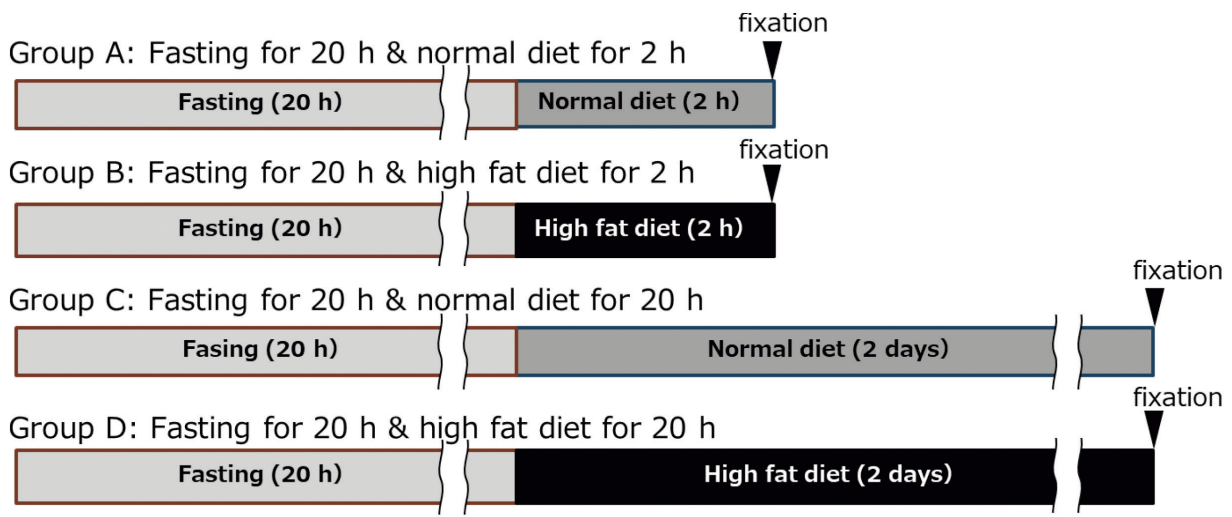

Fig. 1 Experimental design. Animals were divided into 4 groups. Group A: animals were fasted for $20 \mathrm{~h}$ followed by feeding of a normal diet for $2 \mathrm{~h}$. Group B: animals were fasted for $20 \mathrm{~h}$ followed by feeding of a high-fat diet for $2 \mathrm{~h}$. Group C: animals were fasted for $20 \mathrm{~h}$ followed by feeding of a normal diet for $2 \mathrm{~d}$. Group D: animals were fasted for $20 \mathrm{~h}$ followed by feeding of a high-fat diet for $2 \mathrm{~d}$.

netron sputtering coater (MPS-10; Vacuum Device Co. Ltd., Ibaraki, Japan), and observed in a scanning electron microscope (S-3700; Hitachi) using the backscattered electron (BSE) mode at an accelerating voltage of $10 \mathrm{kV}$.

Statistical analysis. BSE-mode SEM images of the villi with complete removal of epithelial covering were used for statistical analysis (1). Each SEM image of a single foliated villus was carefully captured after the flat surface was positioned horizontally with respect to the imaging surface. The number and area of fenestrations as well as the area of the epithelial basal lamina were then measured from these SEM images using ImageJ software (https:// imagej.nih.gov/ij/) as follows.

First, binary images were created from the original BSE-mode SEM images using the ImageJ software. Since the fenestrations appeared dark against the bright surface of the basal lamina in the BSEmode SEM images, almost all fenestrations were automatically extracted from these binary images. The misjudged fenestrations were then carefully corrected manually using the editing software Photoshop (Photoshop CS 5; Adobe Systems Inc., CA, USA). In these images, fenestrations associated with free cells were colored green, and the other fenestrations were colored red (see Fig. 5). Free cells passing through the fenestrations were manually marked in blue. These colored images were processed again using ImageJ to calculate 1) the number of fenestrations per unit villus area, 2) the proportion of the total fenestration area to the villus area, and 3) the proportion of fenestrations with free cell attachment to the total number of fenestrations.
In order to avoid preparation artifacts (e.g., shrinkage or distortion of the villi), the number and area of fenestrations were calculated in the middle $60 \%$ of the entire height of each villus. Mean and SD were calculated using Microsoft Excel (Microsoft Corporation, Redmond, WA). Student's $t$-test was used for comparison between groups and p-values of less than 0.05 were considered statistically significant.

\section{RESULTS}

\section{Light and electron microscopy}

Light microscopy of toluidine-blue stained semithin sections revealed the localization of chylomicrons or lipid droplets absorbed in the villi of upper jejunum since the round lipid droplets were strongly stained with toluidine blue (Fig. 2). Numerous lipid droplets were found in the apical cytoplasm of the epithelial cells of the villi after feeding the high-fat diet for $2 \mathrm{~h}$ (Fig. 2b). Chylomicrons were also found in the intercellular space of the epithelium and were also dispersed in the lamina propria of the villi. Lipid droplets in the epithelial cells decreased in the intestinal villi after feeding the high-fat diet for $2 \mathrm{~d}$, but chylomicrons were dispersed both in the intercellular spaces of the epithelium and the lamina propria (Fig. 2d).

Chyromicrons in relation to the ultrastructure of the villus in the semithin sections were more clearly observed under the BSE mode of the SEM (Fig. 3) and by TEM of ultrathin sections (Fig. 4). In some places of the intestinal villi, chylomicrons accumulated in the intercellular space of the basal side of the epithelium after feeding the high-fat diet for $2 \mathrm{~h}$ 

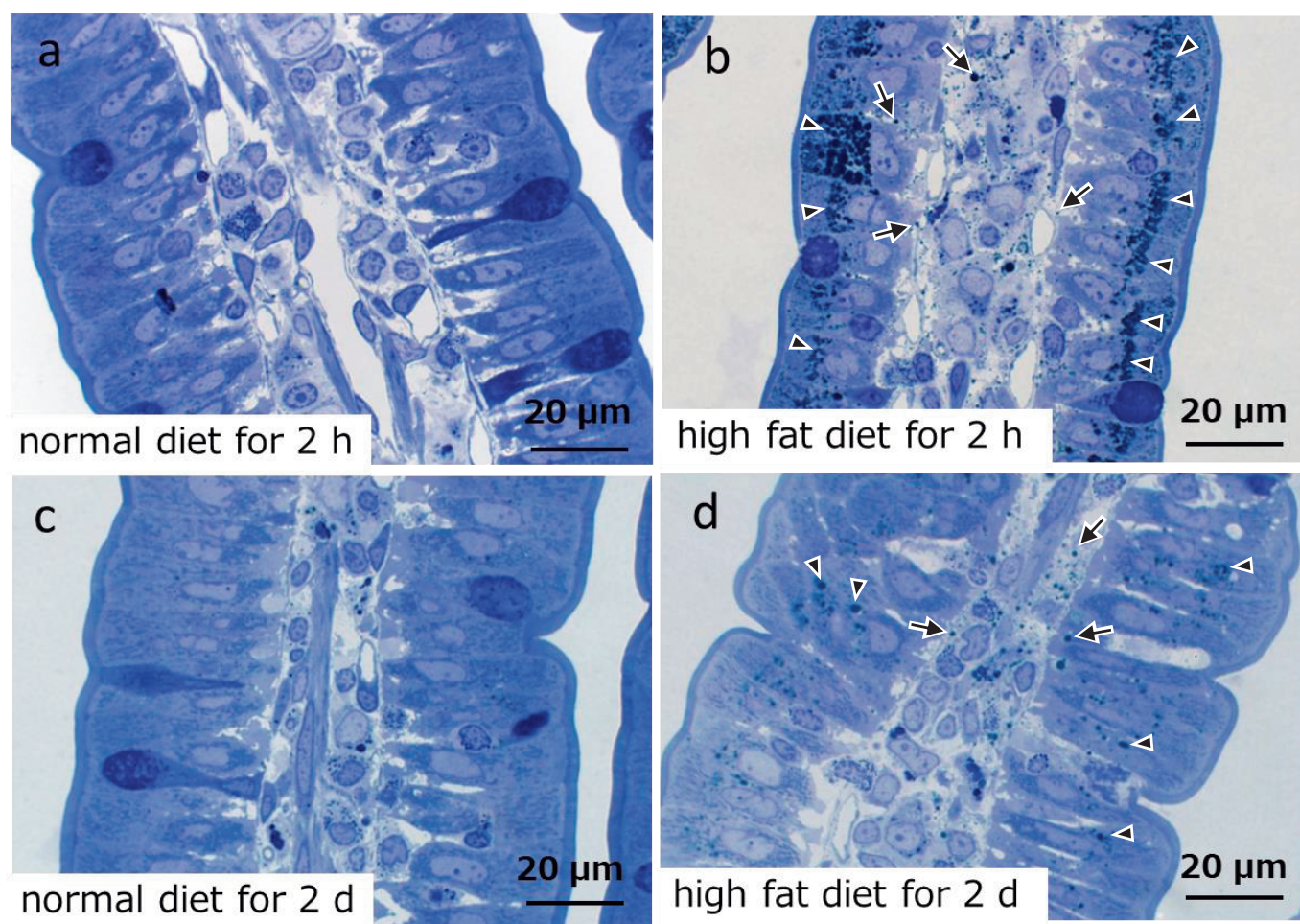

Fig. 2 Light micrographs of toluidine blue stained semithin sections of the intestinal villi in the rat upper jejunum (a. normal diet for $2 \mathrm{~h}, \mathbf{b}$. high-fat diet for $2 \mathrm{~h}$, c. normal diet for $2 \mathrm{~d}$, and $\mathbf{d}$. high-fat diet for $2 \mathrm{~d}$ ). Arrowheads indicate lipid droplets in the epithelial cells, and arrows indicate chylomicrons both in the epithelium and lamina propria.

(Fig. 3c). The size of chylomicrons was larger in the basal side than in the apical side of the epithelium, and it was up to about $4 \mu \mathrm{m}$ in diameter. Chylomicrons were also found in the lamina propria beneath the epithelial basal lamina but they were less than $1 \mu \mathrm{m}$ in diameter (Fig. 4). Migrating leukocytes such as lymphocytes and eosinophils were observed in the intracellular space of the epithelium in every group.

\section{SEM of macerated/ultrasonicated tissues}

Since osmium maceration with ultrasonic treatment was useful for removing the epithelial cells from the villi of the specimen (1), the entire surface of the villous basal lamina could be directly observed under SEM (Figs. 5 and 6). These SEM images clearly showed that the villous basal lamina had numerous fenestrations. These fenestrations could be divided into three types: fenestrations without free cells, fenestrations associated with free cells, and fenestrations covered with migrating free cells (Fig. 5). The number and size of those fenestrations were changed in response to a high-fat diet; free cells adhering to the fenestrations seemed to increased two hours after feeding high-fat diet, while the size of the fenes- trations without free cells apparently increased after feeding high-fat diet for $2 \mathrm{~d}$ (Fig. 6)

\section{Number of basal lamina fenestrations in a villus (Fig. 7)}

Since the shape of the villus in the rat upper jejunum was foliated, the surface area of one side of each villus could be easily calculated from the SEM images. We thus calculated the surface area and the number of fenestrations in the middle region $(60 \%)$ of each villus (Fig. 5a) and compared the number of fenestrations per unit villous area between the normal diet groups (groups A and C) and the high-fat diet groups (groups B and D). The average number per unit area was $9.7 \times 10^{2} / \mu \mathrm{m}^{2}$ in group $\mathrm{A}$ (normal diet fed for $2 \mathrm{~h}$ ), $9.17 \times 10^{2} / \mu \mathrm{m}^{2}$ in group B (highfat diet fed for $2 \mathrm{~h}$ ), $9.38 \times 10^{2} / \mu^{2}$ in group $\mathrm{C}$ (normal diet fed for $2 \mathrm{~d}$ ), and $10.3 \times 10^{2} / \mu^{2}$ in group D (high-fat diet fed for $2 \mathrm{~d}$ ). Statistical analysis showed that no significant difference was found between the normal-diet and the high-fat diet fed groups. 

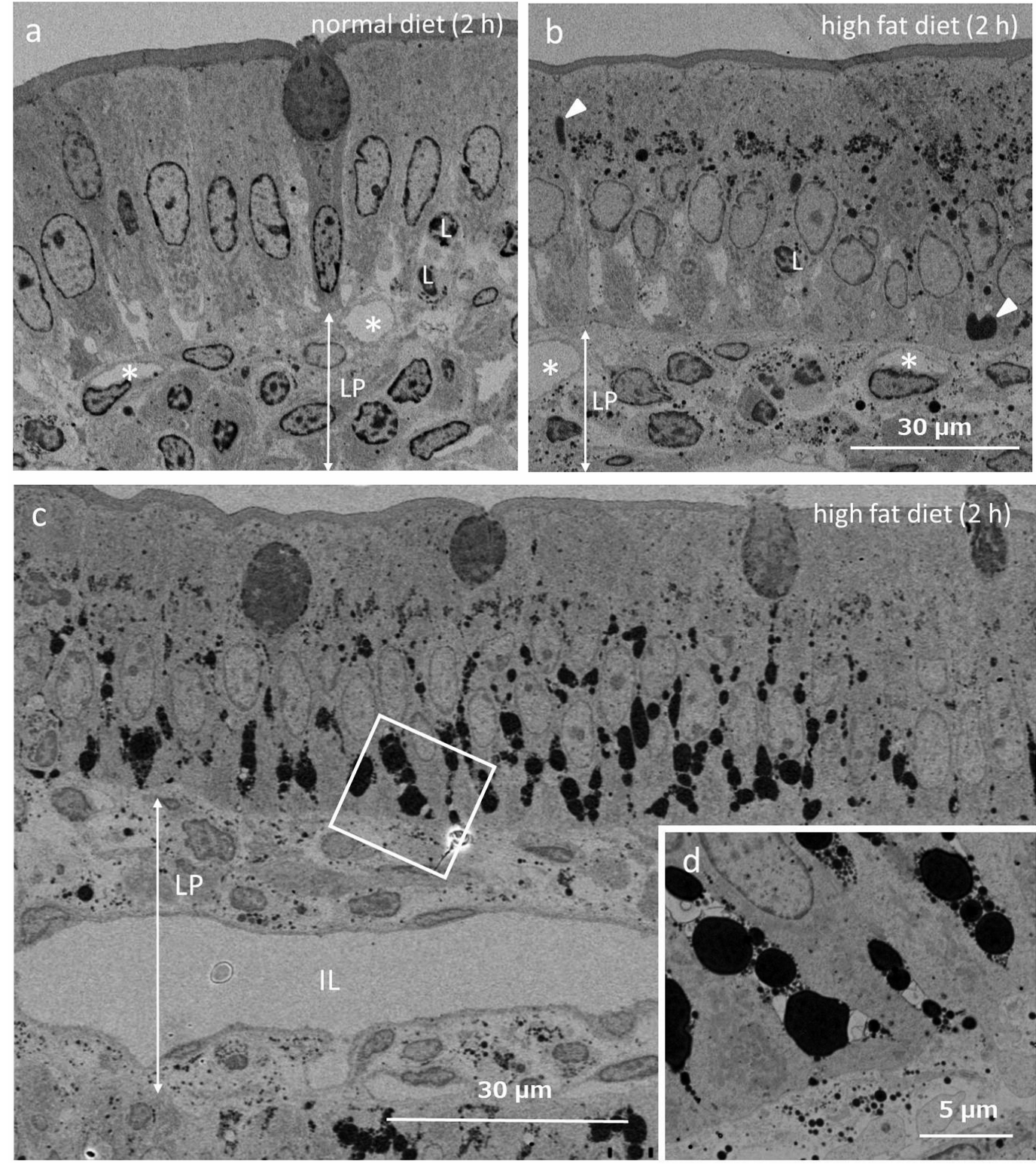

Fig. 3 BSE-mode SEM images of semithin sections of the intestinal villi (a. normal diet for $2 \mathrm{~h}$, $\mathbf{b}-\mathbf{d}$. high-fat diet for $2 \mathrm{~h}$ ). LP: lamina propria, L: migrating leukocyte, ${ }^{*}$ blood capillary. IL: initial lymphatic. Arrowheads in $\mathbf{b}$ indicate chylomicrons in the intracellular space of the epithelium. $\mathbf{d}$ is a closer view of the white box in $\mathbf{c}$.

The total area of basal lamina fenestrations in a villus (Fig. 8)

To compare the total area of basal lamina fenestrations in a villus between the normal and the high-fat diet fed groups, we calculated the proportion of the total fenestration area to a single villus area in each group. This proportion was 3\% in group A (normal diet fed for $2 \mathrm{~h}$ ), $3 \%$ in group B (high-fat diet fed for $2 \mathrm{~h}$ ), $2.83 \%$ in group $\mathrm{C}$ (normal diet fed for $2 \mathrm{~d}$ ), and $3.8 \%$ in group D (high-fat diet fed for $2 \mathrm{~d}$ ). No significant difference was found between the normal and the high-fat diet fed groups after $2 \mathrm{~h}$ of feeding but the fenestration area was significantly larger in the high-fat diet fed group than in the normal-diet fed group after feeding for $2 \mathrm{~d}(P<0.01)$. Our experiments also showed that fenestration area was not different in the two normal-diet fed groups of $2 \mathrm{~h}$ and $2 \mathrm{~d}$ feeding, while the area was significantly different in two high-fat diet fed groups of $2 \mathrm{~h}$ and $2 \mathrm{~d}$ feeding. Small fenestrations of less than about $4 \mu \mathrm{m}^{2}$ decreased and larger fenestrations of more than $6 \mu \mathrm{m}^{2}$ increased in the $2 \mathrm{~d}$ high-fat diet fed group 

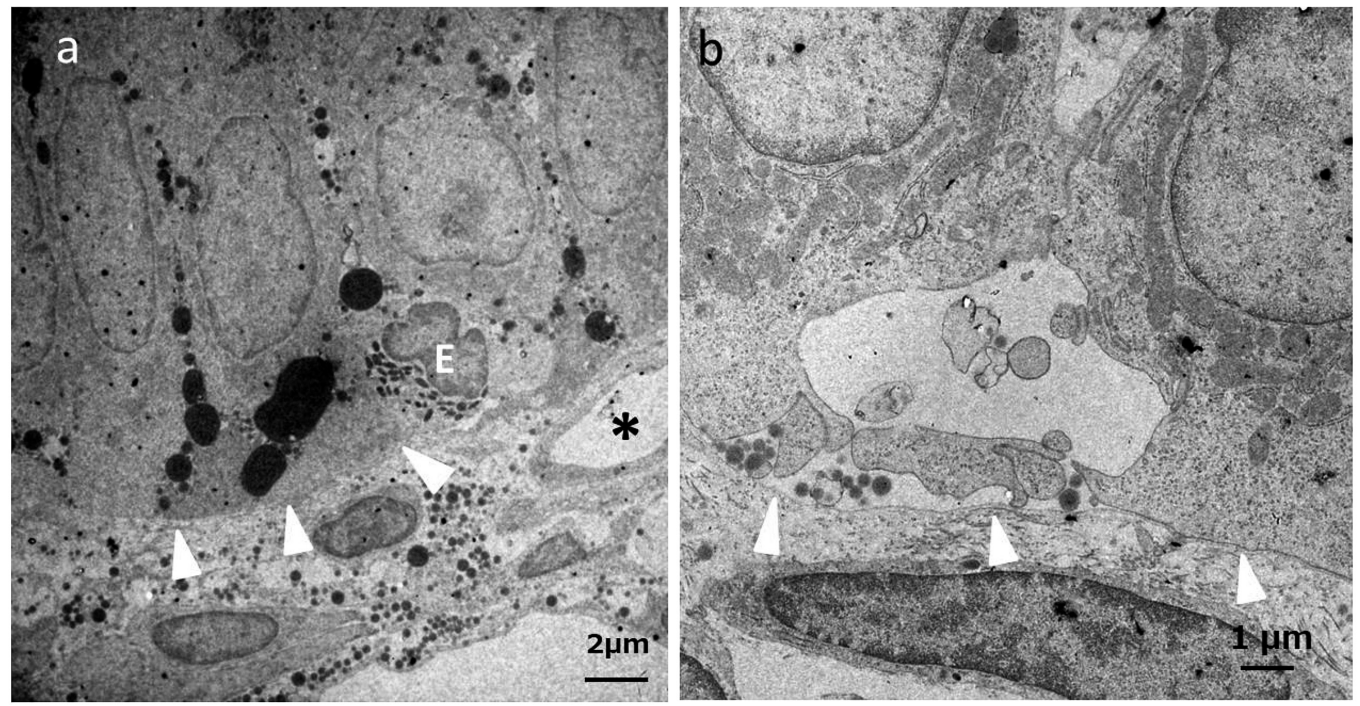

Fig. 4 TEM images of ultrathin sections of a part of the intestinal villi. Arrowheads indicate the basal lamina of the villous epithelium. E: eosinophil, ${ }^{*}$ blood capillary in the lamina propria.
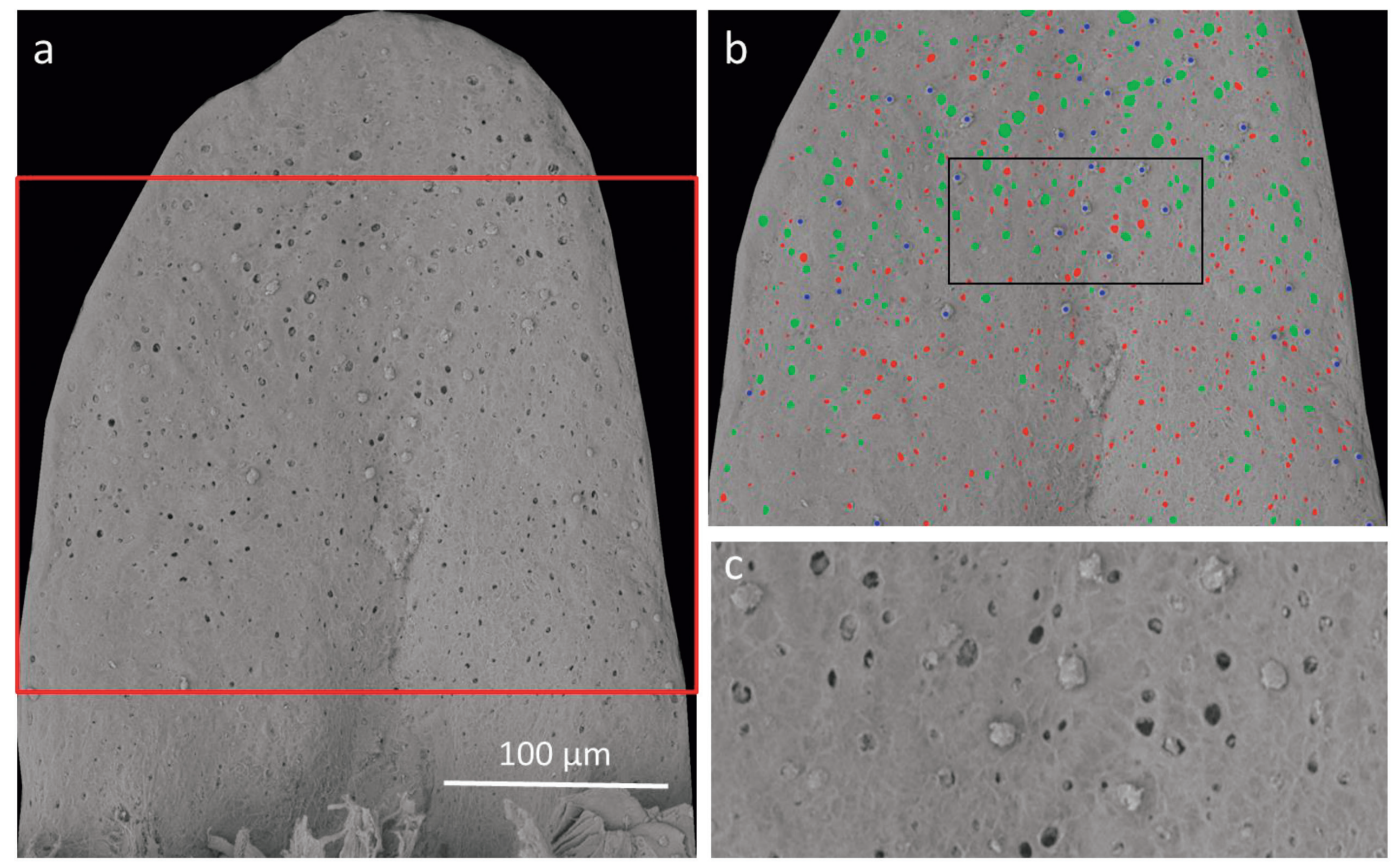

Fig. 5 SEM images of an intestinal villus found in the upper jejunum of the high-fat diet group (2 $\mathrm{h}$ feeding). For statistical analysis, BSE images at an accelerating voltage of $10 \mathrm{kV}$ (a) were used because fenestrations were observed as dark spots compared with the bright surface of the basal lamina. As shown in $\mathbf{b}$ and $\mathbf{c}$ (closer view of the framed area of $\mathbf{b}$ ), fenestrations without free cells are colored red, and those associated with free cells are colored green. Free cells passing through fenestrations are also indicated as blue dots. Calculations were made in the middle $60 \%$ of the entire height of each villus (framed by the red square of $\mathbf{a}$ ).

(Fig. 9).

Number of free cells passing through the basal lamina fenestrations (Fig. 10)

The number of free cells passing through fenestra- tions was compared between the normal diet groups $(\mathrm{A}$ and $\mathrm{C})$ and the high-fat diet groups (B and D). The percentage of the migrating cells per total number of fenestrations was $1.44 \%$ at $2 \mathrm{~h}$ and $1.28 \%$ at $2 \mathrm{~d}$ in the normal-diet fed groups, while it was 

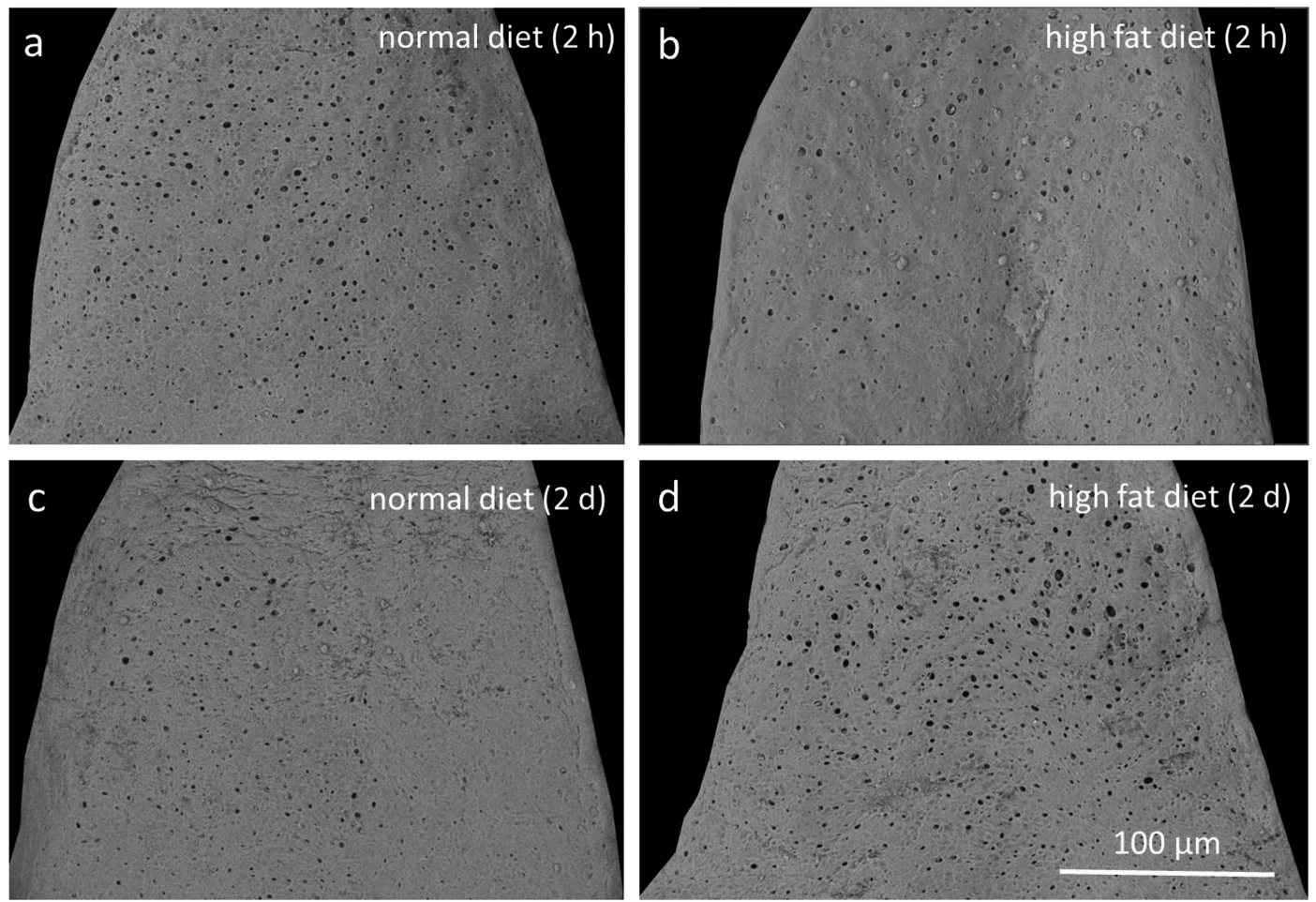

Fig. 6 SEM images of the basal lamina of the intestinal villi in the upper jejunum. a. normal diet for $2 \mathrm{~h}$, $\mathbf{b}$. high-fat diet for $2 \mathrm{~h}$, c. normal diet for $2 \mathrm{~d}$, d. high-fat diet for $2 \mathrm{~d}$. Free cells appear to increase in the high-fat diet groups for $2 \mathrm{~h}$ (b) and size of fenestrations appears to increase in the fatty diet group for $2 \mathrm{~d}$.

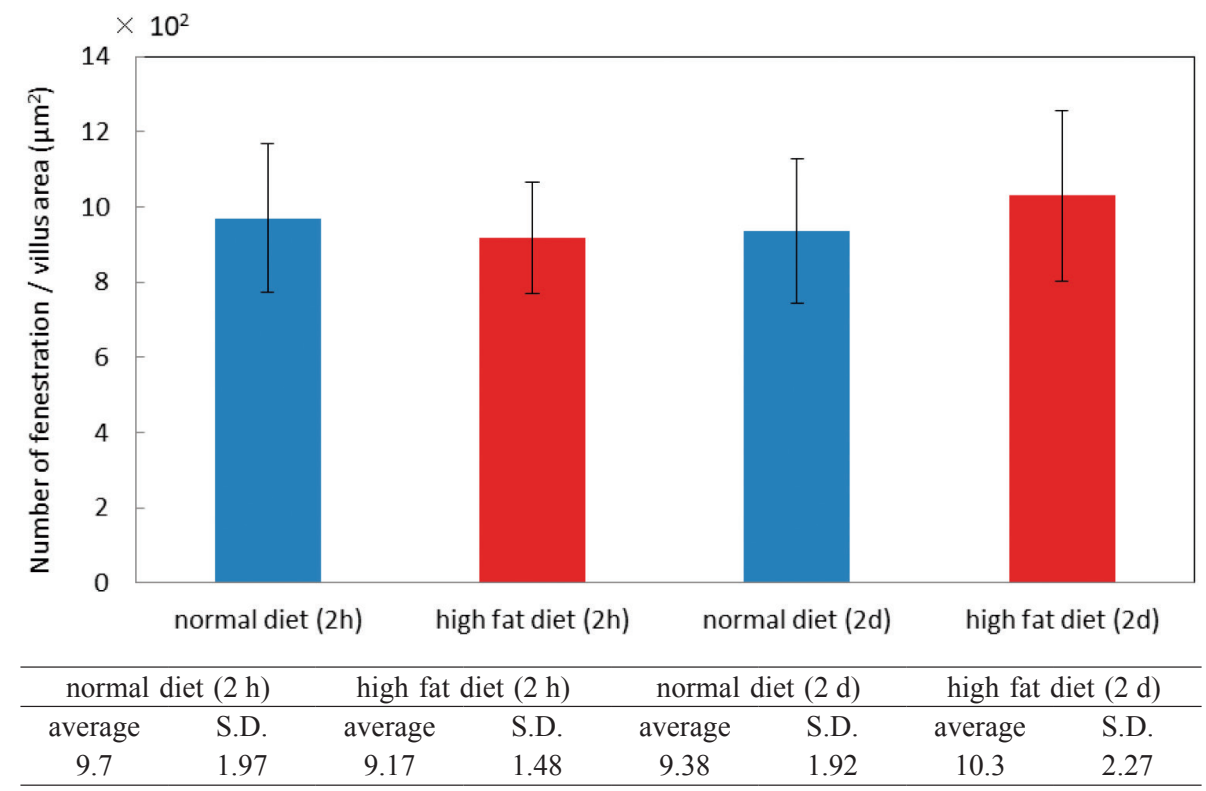

Fig. 7 Number of fenestrations per unit area of the villus. No significant difference was found between each two groups.

$4.86 \%$ at $2 \mathrm{~h}$ and $0.94 \%$ at $2 \mathrm{~d}$ in the high-fat diet fed groups. Thus, the number of the free cells was significantly greater in the high-fat diet fed group than in the normal-diet fed group at $2 \mathrm{~h}(P<0.01)$. 


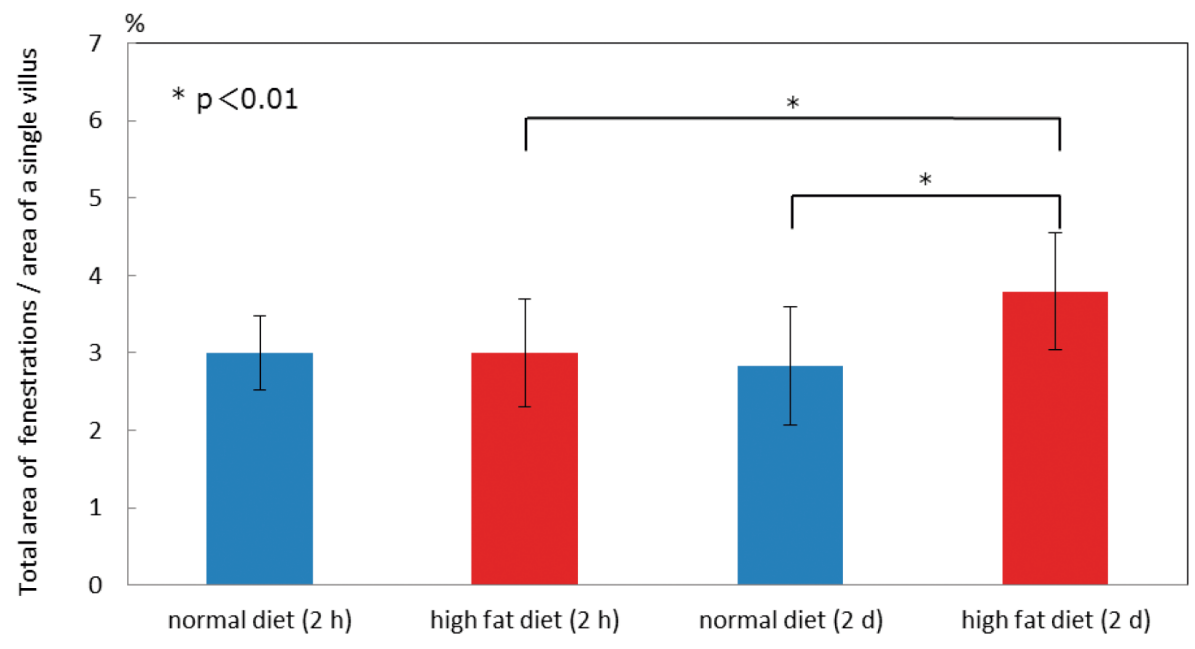

\begin{tabular}{cccccccc}
\hline \multicolumn{2}{c}{ normal diet $(2 \mathrm{~h})$} & \multicolumn{2}{c}{ high fat $\operatorname{diet}(2 \mathrm{~h})$} & \multicolumn{2}{c}{ normal diet $(2 \mathrm{~d})$} & \multicolumn{2}{c}{ high fat $\operatorname{diet}(2 \mathrm{~d})$} \\
\hline average & S.D. & average & S.D. & average & S.D. & average & S.D. \\
3 & 0.48 & 3 & 0.69 & 2.83 & 0.76 & 3.8 & 0.76 \\
\hline
\end{tabular}

Fig. 8 The percentage of the area of fenestrations to villous area. The size of fenestrations significantly increased in the high-fat diet (2 d) group.

high-fat diet (2 h) vs high-fat diet (2 d)

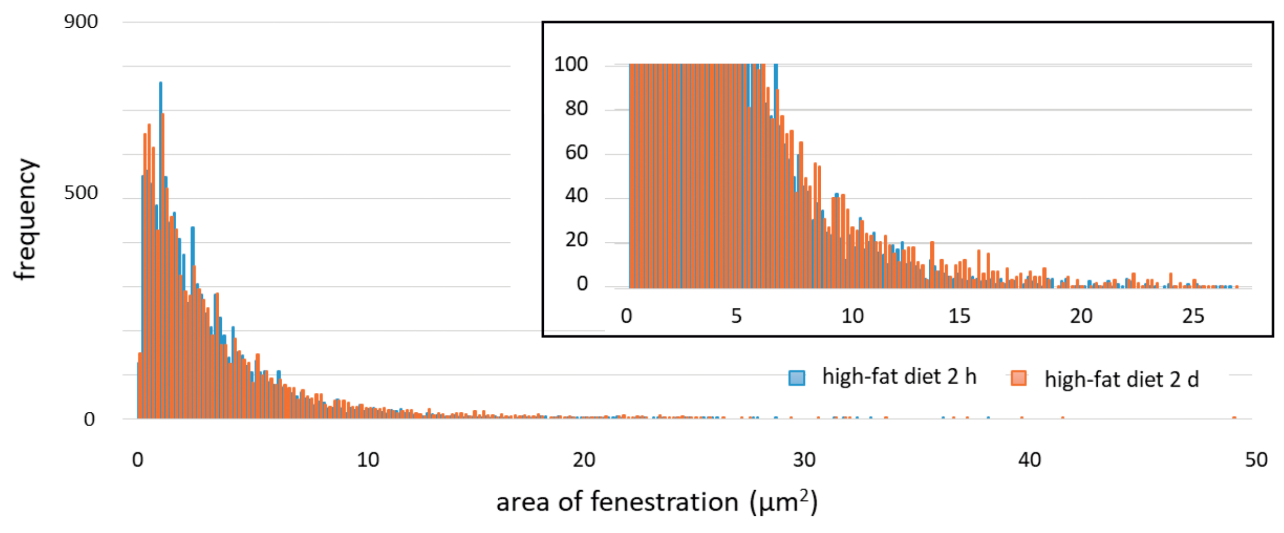

normal diet (2d) vs high-fat diet (2d)

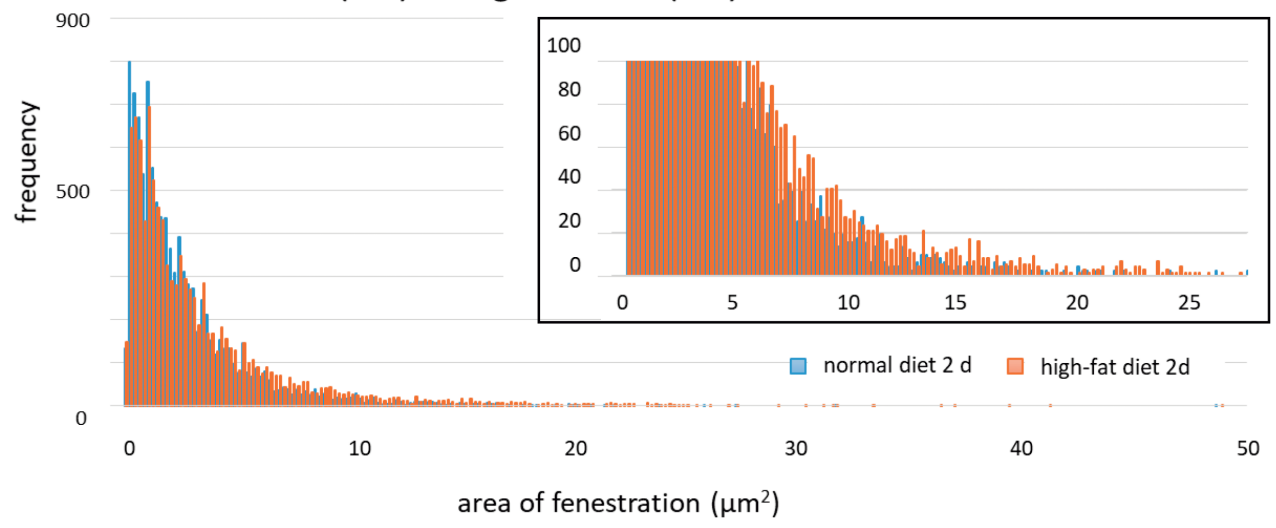

Fig. 9 Histograms showing the size (area) of fenestrations in the normal and high-fat diet groups for $2 \mathrm{~d}$ feeding (upper histogram), and in the fatty groups for $2 \mathrm{~h}$ and $2 \mathrm{~d}$ (lower histogram). Each inset indicates a part of the histogram. 


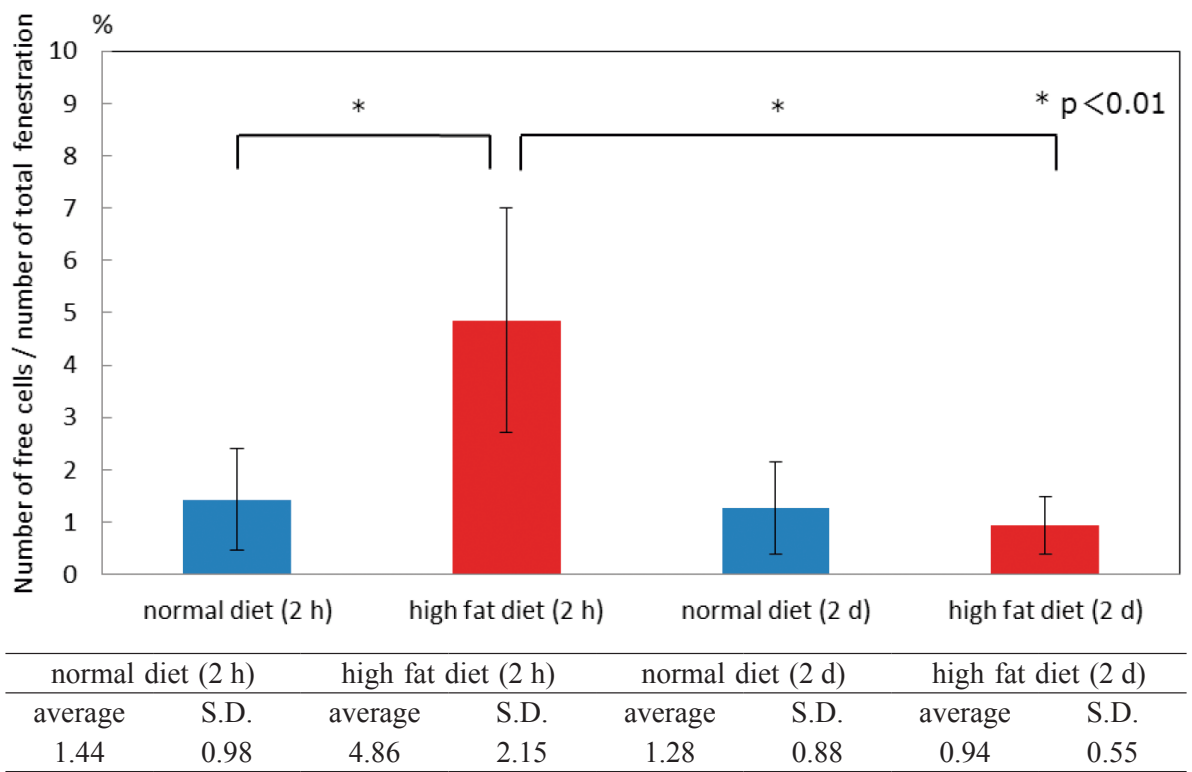

Fig. 10 The percentage of the number of free cells to the total number of fenestrations. Free cells significantly increase in the high-fat diet group for $2 \mathrm{~h}$ feeding.

\section{DISCUSSION}

The present study was performed to clarify changes in size and number of basal lamina fenestrations with changes in the dietary conditions. We observed the structure of the basal lamina in both the normal and high-fat diet fed groups by SEM of osmium macerated/ultrasonicated tissues in the rat upper jejunum, because fat absorption is completed mainly in the jejunum (9). Our previous studies showed that the basal lamina fenestrations were changed through the dynamics of migrating leukocytes due to changes in dietary conditions (1). We then suggested that the dynamics of the fenestration may be related to the regulation of nutrient absorption, particularly lipid absorption, because fenestrations act as a route for large molecules to pass from the intercellular space of the epithelium to the free space of the lamina propria. Our findings also showed the presence of larger chylomicrons (up to about $4 \mu \mathrm{m}$ in diameter) in the epithelium. Although the size of some chylomicrons are much larger than the size of the basal lamina fenestrations, we consider that the larger chylomicrons can be easily fragmented into smaller particles to pass through the fenestrations into the space of the lamina propria.

Our previous studies also showed that migrating leukocytes increased in the upper jejunum after $2 \mathrm{~h}$ of feeding (1). The present study further revealed that the migrating cells showed a higher increase in the high-fat diet fed group than the normal-diet fed group. We also demonstrated that the fenestration size (i.e., area) in the villi also increased after $2 \mathrm{~d}$ of high-fat diet feeding. These findings indicated that high-fat diet feeding induces the migration of free cells into and/or from the epithelium in early stages, resulting in the enlargement of basal lamina fenestrations. The reason for this higher migration of leukocytes in the jejunum in response to high-fat diet is still unclear, though some investigators pointed out the presence of nutrient-induced inflammation in the intestine $(6,4,12)$. The reason for higher migration of leukocytes especially in early stage of highfat diet is also enigmatic. Further studies are required to clarify this in relation to the immune response of intraepithelial migration of leukocytes $(5,18)$.

In this study, we visualized the localization of chylomicrons in the villi by SEM of semithin sections and TEM of ultrathin sections. We showed that chylomicrons often accumulate in the intercellular space of the epithelium delineated by the epithelial basal lamina after $2 \mathrm{~h}$ of high-fat diet feeding. This finding indicates that basal lamina forms a barrier between the intercellular space of the epithelium and the free space of the lamina propria. Basal lamina fenestrations thus provide important pathways for large nutrients including chylomicrons to be released from the epithelial side to the lamina propria.

The present study further provides the idea that efficiency of lipid absorption may be increased by 
high-fat diet feeding for a longer period because basal lamina fenestrations were enlarged due to the high-fat diet. Recent studies revealed that high-fat diet induces various pathophysiological changes and that inflammation also occurs during the development of obesity (10). Types of dietary fat, saturated versus unsaturated, may be also important for understanding the effect of high-fat diets in relation to the development of obesity and metabolic disorders (2). In this study, we used corn oil as a high-fat diet. Since corn oil is a polyunsaturated fat, studies on the effect of other types of fat such as saturated fat, may be also required to clarify the mechanism of lipid absorption in metabolic disorders.

In conclusion, we investigated the structural change in basal lamina fenestrations of rat intestinal villi due to a high-fat diet. This study may provide newer insights in the field of nutrient absorption, especially lipid absorption, in obesity.

\section{REFERENCES}

1. Azumi R, Morita K, Mizutani Y, Hayatsu M, Terai S and Ushiki $T$ (2018) Dynamics of basal lamina fenestrations in the rat intestinal villous epithelium in response to dietary conditions. Biomed Res (Tokyo) 39, 65-74.

2. de Wit N, Derrien M, Bosch-Vermeulen H, Oosterink E, Keshtkar S, Duval C, de Vogel-van den Bosch J, Kleerebezem M, Müller M, and van der Meer R (2012) Saturated fat stimulates obesity and hepatic steatosis and affects gut microbiota composition by an enhanced overflow of dietary fat to the distal intestine. Am J Physiol Gastrointest Liver Physiol 303, G589-G599.

3. Green PHR and Glickman RM (1981) Intestinal lipoprotein metabolism. J Lipid Res 22, 1153-1173.

4. Hara Y, Miura S, Komoto S, Inamura T, Koseki S, Watanabe C, Hokari R, Tsuzuki Y, Ogino T, Nagata H, Hachimura S, Kaminogawa S and Ishii H (2003) Exposure to fatty acids modulates interferon production by intraepithelial lymphocytes. Immunol Lett 86,139-148.

5. Ishikawa $H$, Naito $T$, Iwanaga $T$, Takahashi-Iwanaga $H$,
Suematsu M, Hibi T and Nanno M (2007) Curriculum vitae of intestinal intraepithelial $\mathrm{T}$ cells: their developmental and behavioral characteristics. Immunol Rev 215, 154-165.

6. Ji Y, Sakata Y and Tso P (2011) Nutrient-induced inflammation in the intestine. Curr Opin Clin Nutr Metab Care 14, 315-321.

7. Koga D, Kusumi S, Shodo R, Dan Y and Ushiki T (2015) High-resolution imaging by scanning electron microscopy of semithin sections in correlation with light microscopy. Microscopy (Oxf) 64, 387-394.

8. Komuro T (1985) Fenestrations of the basal lamina of intestinal villi of the rat. Scanning and transmission electron microscopy. Cell Tissue Res 239, 183-188.

9. Kawano S, Sanada Y, Chiba M, Nakagami T and Toki A (2010) Effect of fat supplementation on the maintenance of gut integrity in elemental diet-fed rats. Eur J Pediatr Surg 20, 399-404.

10. Lee CY (2013) The effect of high-fat diet-induced pathophysiological changes in the gut on obesity: what should be the ideal treatment? Clin Transl Gastroenterol 4, e39.

11. McClugage SG and Low FN (1984) Microdissection by ultrasonication: porosity of the intestinal epithelial basal lamina. Am J Anat 171, 207-216.

12. Miura S, Tsuzuki Y, Hokari R and Ishii H (1998) Modulation of intestinal immune system by dietary fat intake: relevance to Crohn's disease. J Gastroenterol Hepatol 13, 1183-1190.

13. Palay SL and Karlin LJ (1959) An electron microscopic study of the intestinal villus. I. The fasting animal. $J$ Biochem Cytol 5, 363-371.

14. Patrick T and Balint JA (1986) Formation and transport of chylomicrons by enterocytes to the lymphatics. Am J Physiol 250, G715-G726.

15. Shimizu M (2010) Interaction between food substances and the intestinal epithelium. Biosc Biotechnol Biochem 74, 232241.

16. Takahashi-Iwanaga H, Iwanaga T and Isayama H (1999) Porosity of the epithelial basement membrane as an indicator of macrophage-enterocyte interaction in the intestinal mucosa. Arch Histol Cytol 62, 471-481.

17. Takeuchi $\mathrm{T}$ and Gonda $\mathrm{T}$ (2004) Distribution of the pores of epithelial basement membrane in the rat small intestine. $J$ Vet Med Sci 66, 695-700.

18. Toner PG and Ferguson A (1971) Intraepithelial cells in the human intestinal mucosa. J Ultrastr Res 34, 329-344. 